\title{
Photothermoelectric and Photoelectric Contributions to Light Detection in Metal-Graphene-Metal Photodetectors
}

\author{
T. J. Echtermeyer, ${ }^{\dagger}$ P. S. Nene, ${ }^{\dagger}$ M. Trushin, ${ }^{\dagger}$ R. V. Gorbachev, ${ }^{\|}$A. L. Eiden, ${ }^{\dagger}$ S. Milana, ${ }^{\dagger}$ Z. Sun, $^{\dagger}$ \\ J. Schliemann, ${ }^{\S}$ E. Lidorikis, ${ }^{\perp}$ K. S. Novoselov, ${ }^{\|}$and A. C. Ferrari ${ }^{*}{ }^{\dagger}$ \\ ${ }^{\dagger}$ Cambridge Graphene Centre, University of Cambridge, Cambridge CB3 0FA, United Kingdom \\ ${ }^{\ddagger}$ Department of Physics, University of Konstanz, D-78457 Konstanz, Germany \\ ${ }^{\S}$ Institute for Theoretical Physics, University of Regensburg, D-93040 Regensburg, Germany \\ ${ }$ School of Physics \& Astronomy, University of Manchester, Oxford Road, Manchester M13 9PL, United Kingdom \\ ${ }^{\perp}$ Department of Materials Science and Engineering, University of Ioannina, Ioannina, 45110, Greece
}

\begin{abstract}
Graphene's high mobility and Fermi velocity, combined with its constant light absorption in the visible to far-infrared range, make it an ideal material to fabricate high-speed and ultrabroadband photodetectors. However, the precise mechanism of photodetection is still debated. Here, we report wavelength and polarization-dependent measurements of metal-graphene-metal photodetectors. This allows us to quantify and control the relative contributions of both photothermo- and photoelectric effects, both adding to the overall photoresponse. This paves the way for a more efficient photodetector design for ultrafast operating speeds.
\end{abstract}

KEYWORDS: Graphene, photodetectors, Raman spectroscopy, photoresponse, optoelectronics

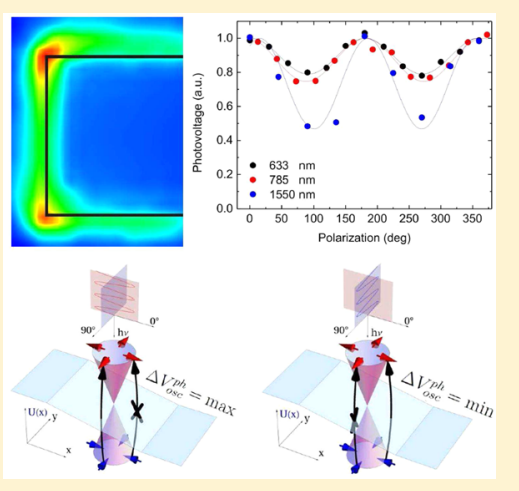

$\mathrm{T}_{\mathrm{n}}^{\mathrm{h}}$ he unique optical and electronic properties of graphene make it ideal for photonics and optoelectronics. ${ }^{1}$ A variety of prototype devices have already been demonstrated, such as transparent electrodes in displays ${ }^{2}$ and photovoltaic modules, ${ }^{3}$ optical modulators, ${ }^{4}$ plasmonic devices, ${ }^{4-9}$ microcavities, ${ }^{10,11}$ and ultrafast lasers. ${ }^{12}$ Among these, a significant effort is being devoted to photodetectors (PDs). ${ }^{6,10,11,13-25}$

Various photodetection schemes and architectures have been proposed to date. The simplest configuration is the metalgraphene-metal (MGM) PD, in which graphene is contacted with metal electrodes as the source and drain. ${ }^{13-18}$ These PDs can be combined with metal nanostructures enabling local surface plasmons and increased absorption, realizing an enhancement in responsivity (i.e., the ratio of the lightgenerated electrical current to the incident light power). ${ }^{6,26}$ Microcavity based PDs were also used, with increased light absorption at the cavity resonance frequency, again achieving an increase in responsivity. ${ }^{10,11}$ Another scheme is the integration of graphene with a waveguide to increase the effective interaction length with light. ${ }^{25,27}$ Hybrid approaches employ semiconducting nanodots as light-absorbing media. ${ }^{22}$ In this case, light excites electron-hole $(\mathrm{e}-\mathrm{h})$ pairs in the nanodots; the electrons are trapped in the nanodot, while the holes are transferred to graphene, thus effectively gating it. ${ }^{22}$ Under applied drain-source bias, this results in a shift in the Dirac point, thus a modulation of the drain-source current. ${ }^{22}$ Due to the long trapping time of the electrons within the dot, the transferred holes can cycle many times through the phototransistor before relaxation and $\mathrm{e}-\mathrm{h}$ recombination. This gives a photoconductive gain; i.e., one absorbed photon effectively results in an electrical current of several electrons. Responsivities $>10^{7} \mathrm{~A} / \mathrm{W}$ were reported, ${ }^{22}$ but with a millisecond time scale, not suitable for, e.g., high-speed optical communications. Devices were also fabricated for detection of $\mathrm{THz}$ light. ${ }^{28,29} \mathrm{In}$ this low energy range, Pauli blocking forbids the direct excitation of $\mathrm{e}-\mathrm{h}$ pairs due to finite doping. Instead, an antenna coupled to source and gate of the device excites plasma waves within the channel. These are rectified, leading to a detectable dc output voltage. ${ }^{28,29}$ PDs based on intrinsic graphene plasmons were also demonstrated. ${ }^{23}$ Graphene, structured into periodic nanoribbons (GNRs), forms a plasmonic metamaterial enabling standing plasmon excitation by infrared light. These lead to an increase of the electron and phonon temperatures, which causes a detectable change of the electrical conductivity of graphene. ${ }^{23}$

MGM-PDs play an important role because they are easy to fabricate, not relying on nanoscale lithography. They operate over a broad wavelength range as the light-matter interaction is mostly determined by graphene itself. Further, ultrahigh

Received: February 6, 2014

Revised: May 29, 2014

Published: June 2, 2014 

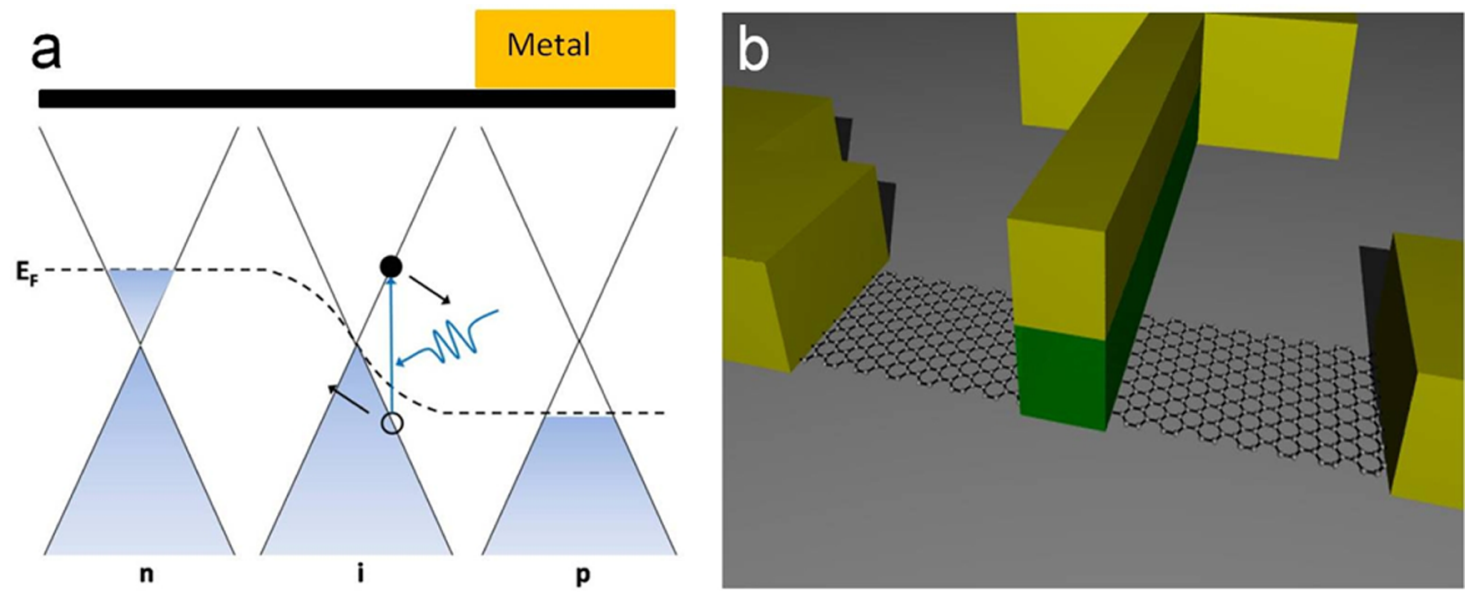

Figure 1. Overview of (a) energy-band profile in MGM-PDs and (b) transistor-like graphene-based PD employing a top gate.

operating speeds can be achieved, ${ }^{16}$ as no bandwidth limiting materials are employed. ${ }^{22}$ MGM-PDs can be considered as the fundamental building block for the other architectures mentioned above. They consist of a graphene channel contacted by two electrodes, either of the same ${ }^{13-15,17,18,21}$ or two different metals. ${ }^{16}$ The difference in work function between the metal pads and graphene leads to charge transfer, ${ }^{30}$ with a consequent shift of the graphene Fermi level in the region below the metal pads. ${ }^{30}$ The Fermi level gradually moves back to that of the uncontacted graphene when crossing from the metal covered region to the metal-free channel. ${ }^{14}$ This results in a potential gradient extending $\sim 100-200 \mathrm{~nm}$ from the end of the metal pad to the metal-free graphene channel. ${ }^{14}$ This inhomogeneous doping profile creates a junction along the channel. This can in principle be a $\mathrm{p}-\mathrm{n}-, \mathrm{n}-\mathrm{n}-$, or $\mathrm{p}-\mathrm{p}$-junction between the graphene underneath and within the channel, as the channel Fermi level can be controlled by the back gate. Figure 1a shows a schematic of the doping profile induced by the metal contact. The formation of this junction is crucially important in the photodetection process, as it results in an internal electric field, capable of separating the light induced e-h pairs.

Another approach to create such junctions, is to exploit a dual-gate transistor structure (Figure $1 b) .{ }^{19,20,31}$ The simultaneous electrostatic doping of the graphene channel by means of a global bottom- and a local top gate allows formation of $n-n-$ $\mathrm{n}, \mathrm{p}-\mathrm{p}-\mathrm{p}, \mathrm{n}-\mathrm{p}-\mathrm{n}$, or $\mathrm{p}-\mathrm{n}-\mathrm{p}$ junctions, respectively. From an application point of view, the dual gate structure requires more fabrication steps, as well as more supply voltages for the gating, but allows control of the doping levels on both sides of the junction. A MGM-PD requires fewer processing steps but has a fixed doping level underneath the contacts, thus allowing fewer operational degrees of freedom. However, it is more suited for applications, due to the simpler fabrication: the single back gate can be used to control the potential gradient in the vicinity of the contacts.

Currently, two effects are thought to contribute to the photoresponse in graphene-based PDs, both requiring spatially in-homogeneous doping profiles: photothermoelectric $^{15,19,20,23,32}$ and photoelectric. ${ }^{13,14,16,18,33}$ The photothermoelectric effect results from local heating of, e.g., the $p-n$ junction, due to the incident laser power. Nonequilibrium hot carriers are excited with an electron temperature $(T)$ higher than that of the lattice. ${ }^{32}$ Different doping levels on both sides of the junction give different Seebeck coefficients. ${ }^{32}$ These are a measure of the induced thermoelectric voltage due to a $T$ gradient and depend on the Fermi-level according to Mott's formula: ${ }^{20,21,32,34}$

$$
S=-\frac{\pi^{2} k_{\mathrm{B}}^{2} T}{3 q} \frac{1}{\sigma} \frac{d \sigma}{d \mu}
$$

with $k_{\mathrm{B}}$ the Boltzmann constant, $\sigma$ the conductivity, $q$ electron charge, and $\mu$ the chemical potential. As a consequence, a net electron flow results, ${ }^{20,21,32,34}$ producing a photothermoelectric voltage $V_{\mathrm{PTE}}::^{20,21,32,34}$

$$
V_{\mathrm{PTE}}=\left(S_{1}-S_{2}\right) \Delta T
$$

with $\Delta T$ the $T$ increase of the hot electrons within the junction, and $S_{1}$ and $S_{2}$ the Seebeck coefficients of the two regions with different doping at the junction.

Due to the nonmonotonous dependence of the difference of the Seebeck coefficients in the two differently doped regions of the junction, the resulting $V_{\mathrm{PTE}}$ exhibits multiple sign reversals in dependence of the gate voltage. ${ }^{20,32}$ This results in a 6-fold pattern, due to the nonmonotonic $S$ in a plot of the photovoltage in dependence of the two doping levels on either side of the junction, as theoretically proposed in ref 32 and experimentally observed in ref 20 .

Besides photothermoelectric effects, light induced heating of one contact can also lead to a $T$ gradient, resulting in a photothermoelectric contribution to the photovoltage, as that described in refs 35 and 36 , where a $T$ gradient was created employing a microfabricated heater. ${ }^{35,36}$

The presence of the junction in the photothermoelectric effect is as important as in the photoelectric effect. The potential gradient within the junction separates the light induced $\mathrm{e}-\mathrm{h}$ pairs and leads to a current flow as in a conventional photodiode. ${ }^{33,37}$ However, to the best of our knowledge, direct evidence and quantification of the photoelectric effect contribution to the photovoltage generation is still missing.

Here we investigate the wavelength and polarization dependent responsivity of MGM-PDs. The measured light polarization dependent responsivity, combined with the spatial origin of the photoresponse obtained from photovoltage maps, allows us to determine the photoresponse mechanisms and quantitatively attribute it to photothermo- and photoelectric effects. 
Our devices are fabricated as follows. Graphene is produced by mechanical exfoliation of graphite (NGS Naturgraphit $\mathrm{GmbH})$ on top of $\mathrm{Si}+\mathrm{SiO}_{2}(300 \mathrm{~nm})^{38,39}$ and its single layer nature confirmed by optical microscopy ${ }^{40}$ and Raman spectroscopy. ${ }^{41,42}$ E-beam lithography is used to define the contacts, followed by e-beam evaporation of the contact metal, consisting of a $4 \mathrm{~nm}$ Ti adhesion layer, and $80 \mathrm{~nm}$ gold Au pads, using liftoff to ensure good mechanical adhesion as well as good electrical contact. Figure 2 shows an optical micrograph of a

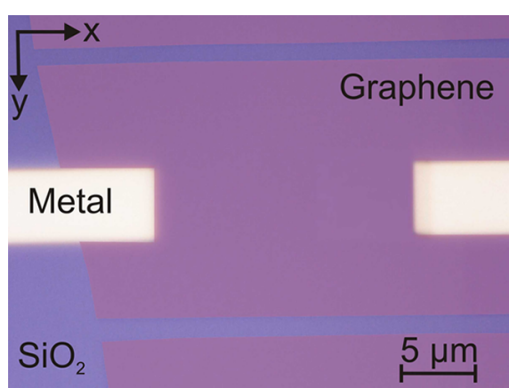

Figure 2. Optical micrograph of device. Graphene is contacted with two metal electrodes.

representative device. The two metal contacts with a width of 5 $\mu \mathrm{m}$ face each other. We use a " $U$ " shaped geometry of the contacts, instead of the more commonly used line-shaped one, since this allow us to monitor the effect of the edges on the device performance. A highly doped $(\rho=0.001-0.005 \Omega \mathrm{cm})$ Si back gate allows us to control the Fermi level in the graphene channel.

Photovoltage mapping is performed at 457, 488, 514, 633, 785 , and $1550 \mathrm{~nm}$ (laser power $P<1 \mathrm{~mW}$ ). The samples are bonded into a chip carrier and connected in a two-terminal configuration to a Keithley Nanovoltmeter 2182A with an additional sourcemeter controlling the gate voltage. The position-dependent generated photovoltage is monitored while a linearly polarized laser light with diffraction limited spot size is scanned over the device. Light from the laser sources is focused through a $100 \times$ ultralong working distance objective (NA $=0.6)$ onto the PDs. A Fresnel-Rhomb polarizer allows us to rotate the light polarization. Polarization control at $1550 \mathrm{~nm}$ is achieved employing a half wavelength plate. Raman measurements are carried out using a Renishaw inVia spectrometer with $P<1 \mathrm{~mW}$ to avoid any possible damage. This allows monitoring defects, ${ }^{42-45}$ as well as local doping. $6,42,46,47$

The doping of the $\mathrm{p}-\mathrm{n}$ junction can be determined by measuring the back gate voltage dependence of the photoresponse. Figure 3a compares the photovoltage in dependence of back gate voltage, $V_{g}$, with the resistance, at an incident light wavelength of $633 \mathrm{~nm}$. The photovoltage shows a sign reversal at $-5 \mathrm{~V}$ relative to the Dirac point, $V_{\mathrm{D}}$. The photovoltage is zero at $V_{\mathrm{g}}-V_{\mathrm{D}}=-5 \mathrm{~V}$, as the doping underneath the metal contact and in the noncontacted graphene is equal, meaning that no junction is present, thus no photovoltage can be produced. The point of vanishing photovoltage lies in the pdoped branch of the resistance curve. From the carrier density $n$ $=\varepsilon_{0} \varepsilon_{\mathrm{SiO}_{2}}\left(V_{\mathrm{g}}-V_{\mathrm{D}}\right) / q t$, with $t$ the oxide thickness, the Fermi level $E_{\mathrm{F}}=\hbar v_{\mathrm{F}}(\pi n)^{1 / 2}$ can be derived to be $\sim 60 \mathrm{meV} .^{38,39}$ This p-doping underneath the metal contact is independent of back gate voltage, due to Fermi-level pinning. ${ }^{30}$ For photovoltage mapping, the back gate voltage is set to $V_{\mathrm{g}}-V_{\mathrm{D}}=+10 \mathrm{~V}$ to
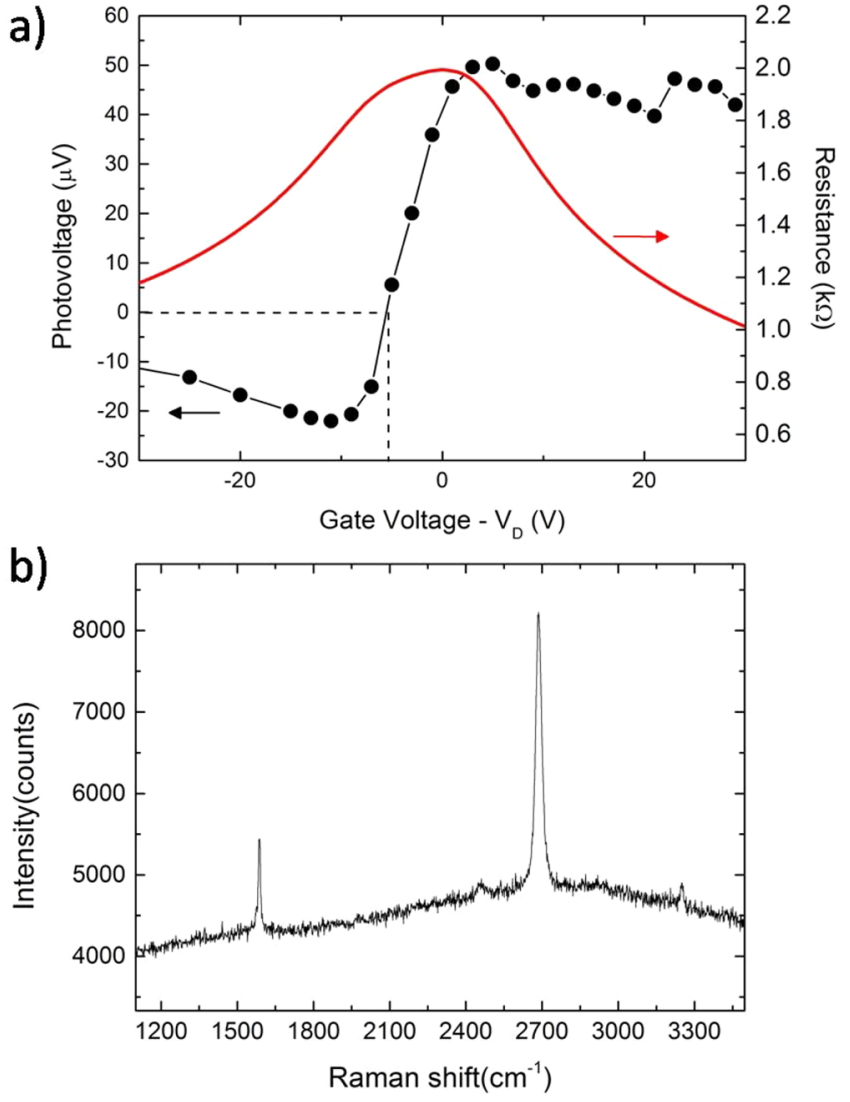

Figure 3. (a) Photovoltage and resistance as a function of back gate voltage. (b) Raman spectrum measured at $514.5 \mathrm{~nm}$ and $V_{\mathrm{g}}-V_{\mathrm{D}}=-5$ $\mathrm{V}$, corresponding to the voltage at which the photovoltage exhibits a sign reversal in a.

achieve $\mathrm{n}$-doping $\sim 100 \mathrm{meV}$ in the noncontacted graphene, thus a $\mathrm{p}-\mathrm{n}$ junction. The doping values are in good agreement with what can be estimated from the Raman spectrum in Figure $3 \mathrm{~b}$, measured at $V_{\mathrm{g}}-V_{\mathrm{D}}=-5 \mathrm{~V}$. The spectrum shows no $\mathrm{D}$ peak, indicating negligible defects. ${ }^{41-44}$ The $2 \mathrm{D}$ peak is a single sharp Lorentzian with full width at half-maximum, FWHM(2D) $\sim 28 \mathrm{~cm}^{-1}$, signature of single layer graphene (SLG). The G peak position, Pos $(\mathrm{G})$, and $\operatorname{FWHM}(\mathrm{G})$, are $\sim 1587 \mathrm{~cm}^{-1}$ and $\sim 9 \mathrm{~cm}^{-1}$. Pos $(2 \mathrm{D}) \sim 2686 \mathrm{~cm}^{-1}$, and the $2 \mathrm{D}$ to $\mathrm{G}$ intensity and area ratios, $I(2 \mathrm{D}) / I(\mathrm{G})$ and $A(2 \mathrm{D}) / A(\mathrm{G})$, are 3.1 and 8.8 , respectively. This indicates p-doping $<100 \mathrm{meV},{ }^{47}$ confirming the electrical characterization. Further, while pristine SLG absorbs $2.3 \%$ of the incident light, ${ }^{67}$ doping can significantly decrease the absorption by Pauli blocking. ${ }^{48,49}$ However, the estimated low doping level derived from the electrical and Raman measurements does not induce any absorption decrease in the wavelength range used in this work.

After confirming that both contacts behave identically by taking a full scan of the device, we will henceforth consider only one of the metal contacts. Figure 4 shows the influence of the excitation wavelength on photovoltage for a $\mathrm{p}-\mathrm{n}$ junction configuration ( $p$-doping of graphene underneath the contact and $\mathrm{n}$-doping in the graphene channel). The photovoltage maps at short and long wavelengths are very different. For 785 $\mathrm{nm}$ excitation (Figure $4 \mathrm{~b}$ ) the photovoltage is mostly generated close to the contact edge where the $\mathrm{p}-\mathrm{n}$ junction is located and vanishes where the graphene flake ends underneath the contact, indicated by the dotted line. At the corners of the metal contact a hot spot of enhanced responsivity occurs, due to curvature 

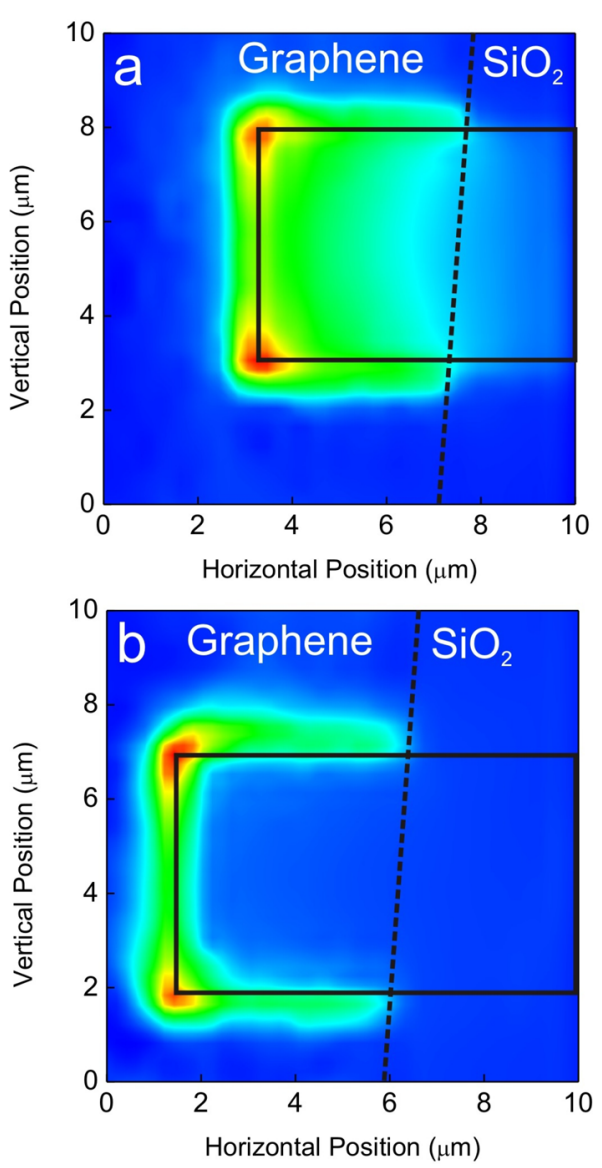

Figure 4. Photovoltage maps for (a) $457 \mathrm{~nm}$ and (b) $785 \mathrm{~nm}$ excitations.

induced electric-field enhancement (lightning-rod effect). ${ }^{50} \mathrm{On}$ the other hand, at $457 \mathrm{~nm}$ (Figure $4 \mathrm{a}$ ) the whole contact area contributes to the photovoltage, with maxima at the contact edges. Even far away from the $\mathrm{p}-\mathrm{n}$ junction located at the edge of the metal contact, a photovoltage is produced. This persists in the metal contact even when graphene is absent underneath, as such extending beyond the indicated dotted line.

Figure 5 shows that at 633,785 , and $1550 \mathrm{~nm}$ a single peak at the contact edge is observed. Wavelengths of 457,488 , and 514 $\mathrm{nm}$ lead to an additional decay of the photovoltage into the metal contact, with increasing decay lengths for shorter wavelengths.

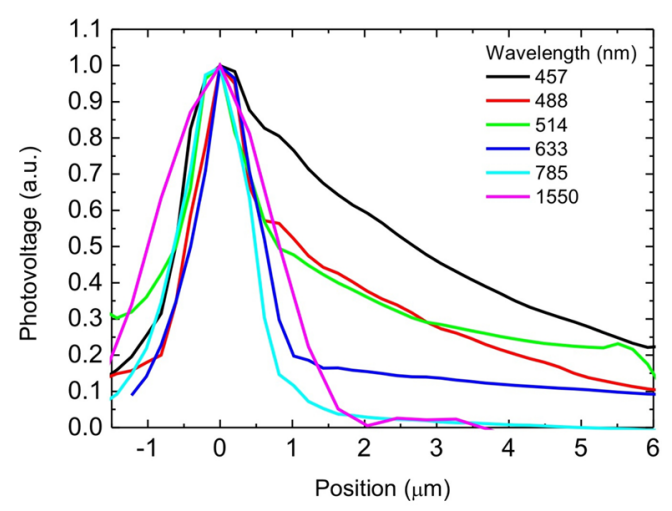

Figure 5. Spatial profile of photovoltage in the center of the metal contact.
We now consider the dependence of the responsivity, g, on excitation wavelength, Figure 6a. This shows an enhancement
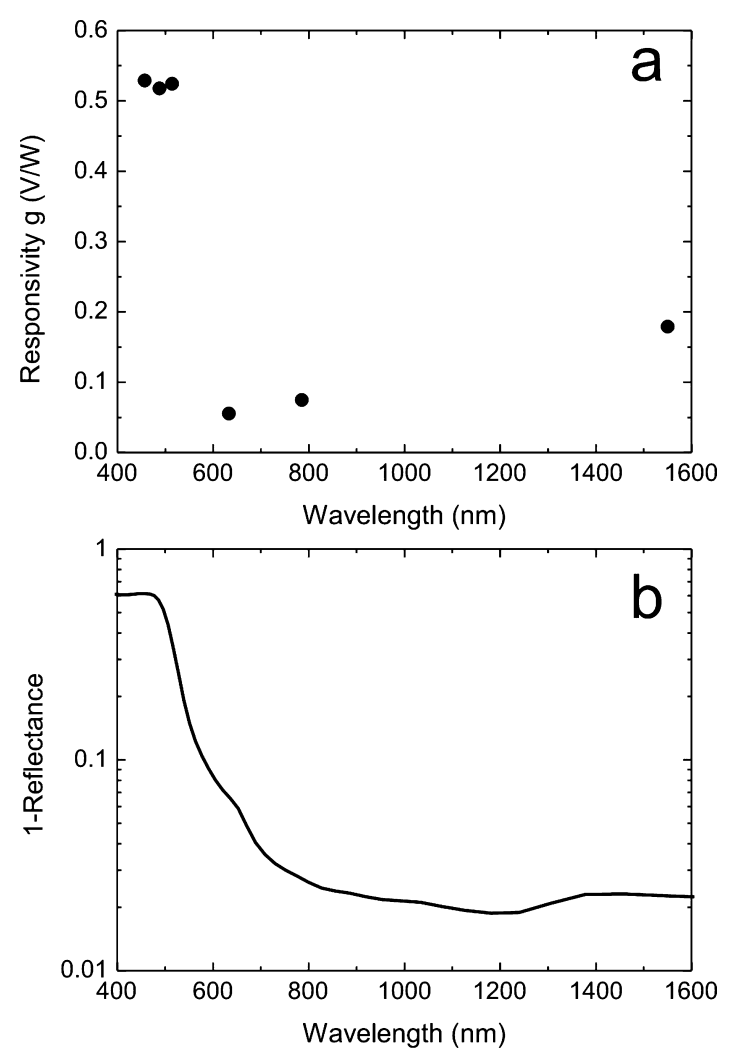

Figure 6. (a) Responsivity of MGM-PD and (b) 1 - reflectance of $\mathrm{Au}$, as a function of excitation wavelength.

toward shorter wavelengths (2.5 times bigger at $457 \mathrm{~nm}$ compared to $1550 \mathrm{~nm}$ ). We assign this to an increased absorption of the incident light at shorter wavelengths on the Au contact. This leads to a $T$ rise on the metal contact, thus heating the pn-junction at the contact edge, producing a thermoelectric contribution to the photovoltage. This is consistent with the photovoltage contribution of the metal contact far away from the contact edge, as seen in Figure 4a, because no light is incident directly on the junction. Considering Au's good $(\sim 300 \mathrm{~W} / \mathrm{mK})^{51}$ and graphene's excellent (up to $\sim 5000 \mathrm{~W} / \mathrm{mK}$ ) ${ }^{52}$ thermal conductivities, we assume that heat is transported to the $\mathrm{p}-\mathrm{n}$ junction from within the metal contact, leading to a $T$ gradient across the device and producing a thermoelectric contribution to the photovoltage. ${ }^{35,36}$ Indeed, the trend in Figure 6a follows that of the heat energy $Q[\mathrm{~J}]$ deposited into the metal by the incident laser. This can be expressed as $Q \sim P_{\text {abs }}=(1-R) P_{\text {input }}{ }^{53}$ with $P_{\mathrm{abs}}[W]$ the absorbed power in the metal film, $R$ the $\mathrm{Au}$ film reflectance, and $P_{\text {input }}[W]$ the incident laser power. Figure $6 \mathrm{~b}$ plots the calculated dependence of $1-R$ as a function of wavelength. For metals, the normal incidence $R$ is ${ }^{54}$

$$
R=\frac{\left(n_{1}-n_{0}\right)^{2}+k_{1}^{2}}{\left(n_{1}+n_{0}\right)^{2}+k_{1}^{2}}
$$

where $n_{0}$ is the refractive index of the entrance medium, and $n_{1}$ and $k_{1}$ are the real and imaginary parts of the complex index of refraction of the absorbing medium. Taking Au's complex index of refraction from ref 55 and considering that the entrance medium is air with $n_{0}=1$, the factor $1-R$, proportional to the 
absorbed heat energy, is in good agreement with Figure 6a. 1 $R$ increases a factor 20 from 1550 to $457 \mathrm{~nm}$ and explains the enhanced responsivity at shorter wavelengths as due to pronounced thermoelectric effects resulting from the $\mathrm{Au}$ contact heating. Even in the absence of graphene underneath the contact, a photoresponse is generated as the $\mathrm{Au}$ film spreads the heat energy toward graphene.

To further investigate the influence of thermo- and photoelectric effects on the overall photovoltage, we perform polarization-dependent measurements. Photovoltage maps are acquired at different polarization angles of the incident light, for a given location at the contact edge. Figure 7 plots the

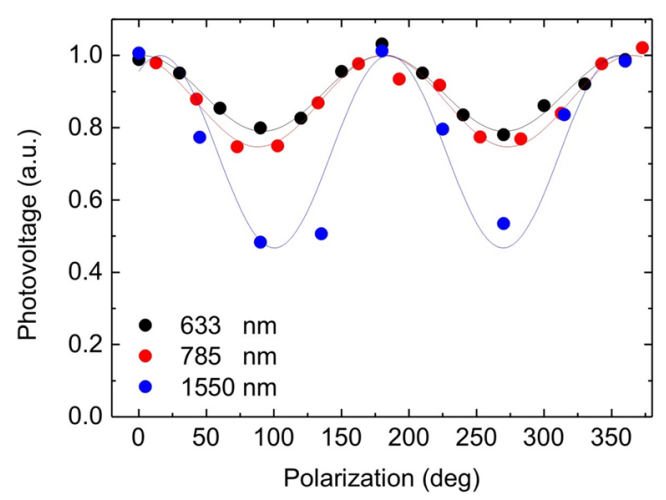

Figure 7. Dependence of photovoltage on incident polarization.

photovoltage in dependence of polarization at 633, 785, and $1550 \mathrm{~nm}$. The convention used is that an angle of $0^{\circ}$ denotes a polarization perpendicular to the metal contact edge. This shows two contributions: one polarization dependent, and another polarization independent.

This behavior could be in principle due to plasmonic effects. ${ }^{6,7}$ Polarization-dependent excitation of plasmons at the metal edge could lead to near-field enhancement, thus a polarization-dependent responsivity enhancement. Raman spectroscopy is used to investigate the influence of the metal electrode on a possible plasmonic dependence on light polarization. Spectra are first taken approaching the metal electrode from the bare graphene in a line scan with $300 \mathrm{~nm}$ steps. Figure 8a plots $I(G)$ as a function of position, for polarization perpendicular to the metal edge (maximum photovoltage). $I(\mathrm{G})$ decreases as the metal edge is approached and no enhancement in the vicinity of the edge is observed, since the metal electrode shields the light. Polarization dependent Raman measurements are then carried out at the metal edge, Figure $8 \mathrm{~b}$. No trend is observed for the angular dependence. Another possible explanation could be surface plasmon polaritons (SPPs) that propagate from within the metal contact toward the junction at the edge of the contact. ${ }^{56}$ However, experiments in combination with theoretical calculations demonstrate that SPPs cannot be excited on a flat metal contact. ${ }^{56}$ Thus, plasmonic effects cannot explain the observed photovoltage angular dependence.

We thus assign the polarization-dependent contribution to the photoelectric effect, due to polarization dependent interband optical excitations. Charge carriers in graphene are the $\pi$-electrons moving on a honeycomb lattice composed of two interpenetrating hexagonal sublattices. The sublattice degree of freedom is commonly associated with the pseudospin, ${ }^{57}$ which relates to the relative amplitude of the
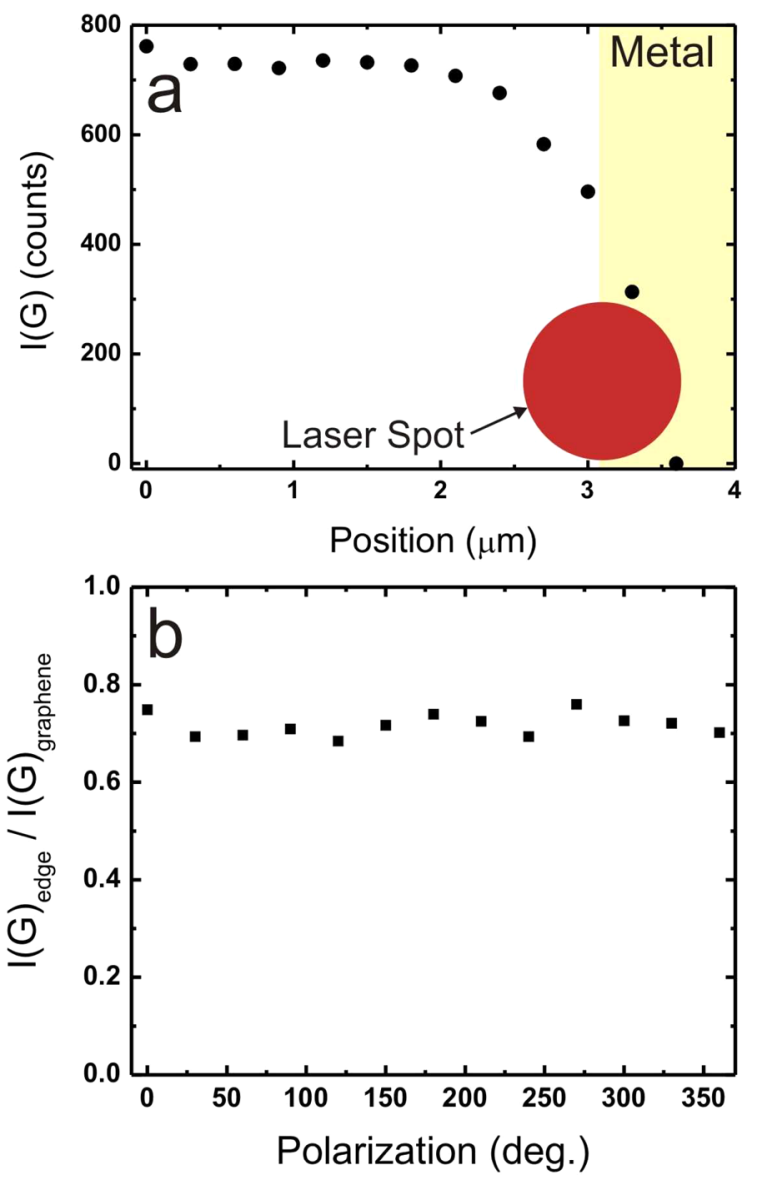

Figure 8. (a) Linescan of $I(G)$ approaching the contact edge. (b) Polarization dependence of $I(\mathrm{G})$ at the edge relative to $I(\mathrm{G})$ away from the edge.

electron wave function located on either sublattice. If all electrons were placed on the "red" sublattice in Figure 9, the

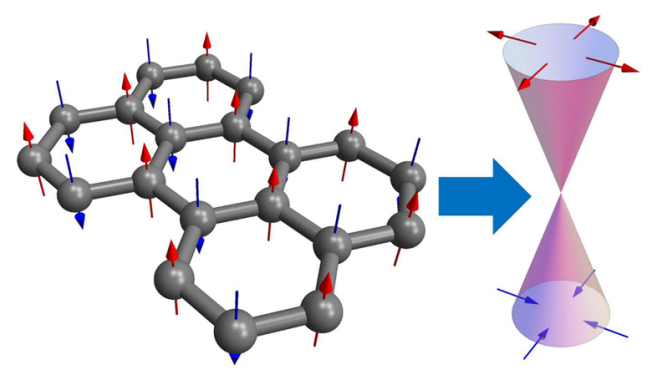

Figure 9. (Left) Honeycomb lattice of graphene and corresponding real space pseudospin orientation of the two interpenetrating Bravais sublattices, denoted in red and blue, respectively, and (right) translation to momentum space.

pseudospin would be pointing upward out of the SLG, whereas electrons on the "blue" sublattice correspond to pseudospin pointing downward out of the SLG. Since both "red" and "blue" lattice sites are occupied by the same carbon atoms, the electron density is distributed equally between these sublattices. Thus, the in-plane pseudospin orientation is determined by the phase difference in the amplitudes on the "red" and "blue" sites. ${ }^{57}$ The resulting texture is radial, as shown in Figure 9.

The pseudospin-locked carriers can be described by the effective Hamiltonian: ${ }^{57} H_{0}=v_{\mathrm{F}} \hat{\sigma} \cdot \mathbf{p}$, where $\mathbf{p}$ is the two- 
component momentum, $\hat{\sigma}$ is the pseudospin operator constructed with the Pauli matrices, and $v_{\mathrm{F}} \approx 10^{6} \mathrm{~ms}^{-1}$ is the Fermi velocity. The pseudospin texture represents the expectation value $\vec{\sigma}$ of $\hat{\sigma}$ with respect to the eigenstates of $\mathrm{H}_{0} \cdot{ }^{57}$

To excite an electron from the valence to the conduction band it is necessary to flip the pseudospin, as it is seen from Figure 9. The interaction Hamiltonian between the charge carriers in graphene and an electromagnetic wave is characterized by the electric $\mathbf{E}=(-1 / c)(\partial \mathbf{A} / \partial t)$ and magnetic $\mathbf{B}=\nabla \times \mathbf{A}$ fields, with $\mathbf{A}$ the vector potential. This can be derived form $H_{0}$ by substituting $\mathbf{p} \rightarrow \mathbf{p}-(q / c) \mathbf{A}: H_{\text {int }}=\left(q v_{\mathrm{F}} /\right.$ c) $\hat{\sigma} \cdot \mathbf{A}$. Assuming a linearly polarized electromagnetic wave with $\mathbf{A}=\mathbf{A}_{0} \cos (\omega t-k z)$, the corresponding electric field is $\mathbf{E}=\mathbf{E}_{0}$ $\sin (\omega t-k z)$, with $\mathbf{E}_{0}=\left(\omega \mathbf{A}_{0} / c\right), \omega=2 \pi c / \lambda$ the radiation frequency, and $k$ the normal component of the wave vector. Considering the commutator $\left[H_{\text {int }}, \hat{\sigma}\right]$, these two operators commute with each other if and only if $\mathbf{A}$ (or $\mathbf{E}$ ) is along $\hat{\sigma}$. The pseudospin is then conserved and interband transitions are forbidden, as for Figure 10a,b. In contrast, $\left[H_{\text {int }}, \hat{\sigma}\right]$ is maximum for $\mathbf{E} \perp \hat{\sigma}$, resulting in an interband transition rate maximum (Figure 10a,b). Note that $\vec{\sigma} \| \mathrm{p}$ because of the pseudospinmomentum locking (Figure 10a,b). As consequence, the photovoltage, $V^{\mathrm{ph}}$, measured on the irradiated junction depends
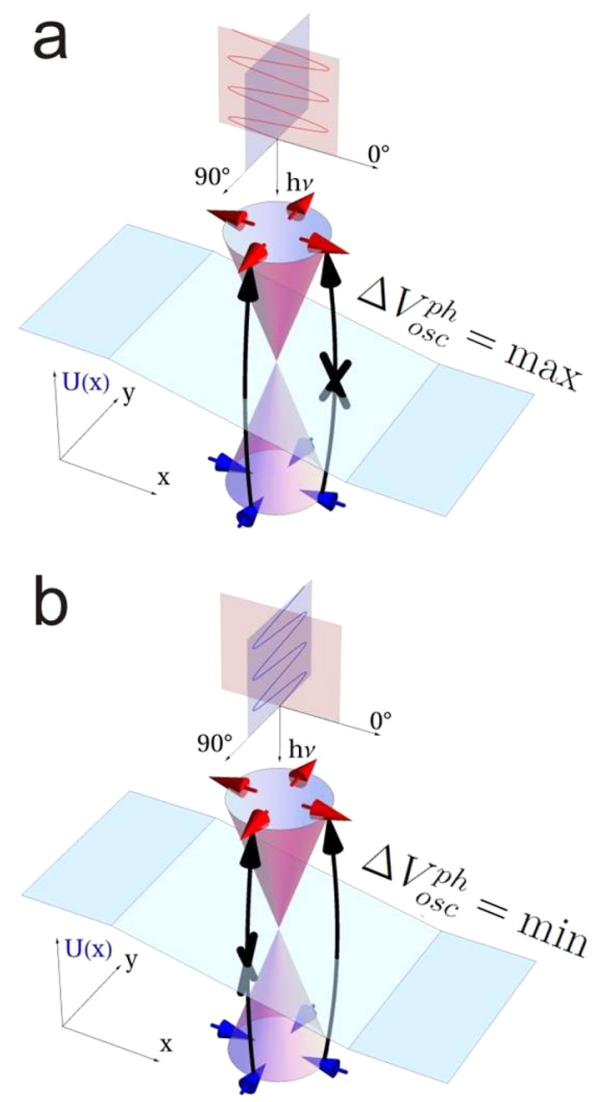

Figure 10. $(\mathrm{a}, \mathrm{b})$ Linearly polarized light incident on a potential step $U(x)$ in graphene and pseudospin-dependent selection rule for interband optical excitations for (a) $\theta_{\mathrm{pol}}=0^{\circ}$; (b) $\theta_{\mathrm{pol}}=90^{\circ}$. The polarization is characterized by the electric field $\mathbf{E}$. The photocarrier generation rate is anisotropic and $\alpha \sin ^{2}(\mathbf{E}, \hat{\mathbf{p}})$, with $\mathbf{p}$ the electron momentum. The driving term of the Boltzmann equation acting on a function of $\mathbf{p} / p$ is maximal when the force $-\nabla U(x)$ is perpendicular to the direction of motion, maximizing the photoresponse at $\theta_{\mathrm{pol}}=0^{\circ}$; see main text. on the relative orientation between the polarization plane of the incident light and the junction.

The relaxation of photoexcited carriers to equilibrium in graphene consists of three processes with three characteristic time scales: ${ }^{12,58-63}$ In the first step, photoexcited carriers lose energy through $\mathrm{e}-\mathrm{e}$ and electron-phonon $(\mathrm{e}-\mathrm{ph})$ scattering on a $\sim 10 \mathrm{fs}$ time scale. ${ }^{58,63}$ Subsequently, this distribution thermalizes through $\mathrm{e}-\mathrm{ph}$ scattering toward a hot Fermi-Dirac distribution, ${ }^{12,58-65}$ with the time scale in the range of hundreds of fs $\left(\tau_{1}\right)^{12,58-63}$ Finally, the hot Fermi-Dirac distribution relaxes to equilibrium by $\mathrm{e}-\mathrm{h}$ recombination, which can lead to plasmon emission, phonon emission, and Auger scattering on a ps time scale $\left(\tau_{2}\right) .{ }^{12,58-63}$

The optical fluence $\Phi$ applied to our devices is low compared to that used in most pump-probe measurements, such as those in refs 58-60 and 66. It is instructive to translate fluence into photoelectron concentration $n^{\mathrm{ph}}=\left(\pi e^{2} / \hbar c\right)(\Phi / \hbar \omega)$, with $\left(\pi e^{2} /\right.$ $\hbar c)=0.023$ the SLG optical absorption, ${ }^{67}$ or, equivalently, $n^{\mathrm{ph}}$ $=\left(\pi e^{2} / \hbar c\right)\left(w_{i} \tau_{2} / \hbar \omega\right)$, where $w_{i}=\left(c\left|\mathbf{E}_{0}\right|^{2}\right) /(8 \pi)$ is the incident radiation power per square. The majority of pump-probe measurements were done for $n^{\mathrm{ph}} \sim 10^{13} \mathrm{~cm}^{-2} .^{58-60,66}$ In our case of continuous wave radiation with laser powers $\sim$ few hundred $\mu \mathrm{W}$ and $\mu \mathrm{m}$ laser spot diameters, we have $n^{\mathrm{ph}} \sim 10^{10}$ $\mathrm{cm}^{-2}$, for a typical total recombination time $\tau_{2} \sim$ ps. The anisotropic distribution function for the photoexcited carriers relaxes to a hot Fermi-Dirac distribution at a $\mathrm{T}$ which could be much higher than room temperature, $T_{\text {room }}{ }^{58,61,62,66}$ In our case, however, the light induced photocarrier concentration is much lower than the always present intrinsic background electron concentration, even at zero chemical potential. The intrinsic e concentration $n$ at $T_{\text {room }}$ can be calculated as $n=$ $4 \int\left(d^{2} k / 4 \pi^{2}\right) f_{+}^{(0)}\left(k, T_{\text {room }}\right)=\left(\pi T_{\text {room }}^{2} / 6 \hbar^{2} v_{\mathrm{F}}^{2}\right)$, with $f_{+}^{(0)}\left(k, T_{\text {room }}\right)$ the electron-Fermi-Dirac distribution, and the multiplier 4 accounting for spin and valley degeneracy. This gives $n \sim 10^{11}$ $\mathrm{cm}^{-2}$, higher than $n^{\mathrm{ph}} \sim 10^{10} \mathrm{~cm}^{-2}$, at the fluence used in our experiments. The same is true for the corresponding hole concentrations $p$ and $p^{\mathrm{ph}}$. The major contribution to the total carrier concentration thus originates from the intrinsic carriers, described by the Fermi-Dirac distribution at $T_{\text {room }}$. In what follows, we therefore assume the characteristic electron temperature to be $T_{\text {room }}$.

Note that the role of carrier-carrier interactions in the relaxation of the photocarrier distribution is reduced substantially since the lower carrier concentration results in less frequent carrier-carrier collisions. As consequence, the ultrafast relaxation time scale $\sim 10 \mathrm{fs}^{58}$ at high fluence is not considered here. We assume the relaxation of the anisotropic photocarrier distribution on a time scale of $\tau_{1}$. This was measured to be $\sim 150-170$ fs. $^{58}$

The interaction between the electromagnetic wave and charge carriers can be quantified using Fermi's golden rule, considering $H_{\text {int }}$ as a perturbation. The steady state distribution function is obtained by balancing the golden-rule $\mathrm{e}-\mathrm{h}$ generation and relaxation rates. Since we are interested in the anisotropic part of the distribution function, the relevant relaxation time is $\tau_{1}=150$ fs discussed above, rather than $\tau_{2}>1$ ps associated with e-cooling and $\mathrm{e}-\mathrm{h}$ recombination. The generation rate is $\alpha \sin ^{2}\left(\theta-\theta_{\mathrm{pol}}\right)$, where $\theta$ is the direction of $\mathrm{e}$ motion with $p_{x}=p \cos \theta, p_{y}=p \sin \theta$, and $\theta_{\mathrm{pol}}$ is the polarization angle; see Methods.

The standard tool for photovoltage calculations is the driftdiffusion equation, ${ }^{68}$ which considers e and $\mathrm{h}$ concentrations, rather than their distribution functions. The angular depend- 
ence of the distribution function is lost when the Boltzmann equation is integrated in momentum space to obtain the driftdiffusion relation. ${ }^{68}$ To retain the angular dependence we have to take one step back and start from the Boltzmann equation:

$$
\mathbf{F} \cdot \nabla_{\mathbf{p}} f_{ \pm}+\mathbf{v} \cdot \nabla_{\mathbf{r}} f_{ \pm}=g_{ \pm}^{\mathrm{ph}}-\frac{\Delta f_{ \pm}}{\tau_{1}}
$$

where $\mathbf{F}$ is the electrostatic force, with $F_{x}=F \cos \phi, F_{y}=F \sin$ $\phi, \mathbf{v}$ is the electron velocity, $g_{ \pm}^{\mathrm{ph}}$ is the photogeneration rate, and $\Delta f_{ \pm}=f_{ \pm}-f_{ \pm}^{(0)}$ is the deviation of the distribution function from the hot Fermi-Dirac distribution $f_{ \pm}^{(0)}$, “ \pm " being the conduction/valence band index. The solution of this equation can be found in Methods. We then calculate the current density $j_{x}=4 \sum_{ \pm} \int\left(\mathrm{d} k^{2} / 4 \pi^{2}\right) v_{x} f_{ \pm}$and set $j_{x}(V)=0$ (open circuit) to extract $V$, which resembles $V^{\mathrm{ph}}$, in the absence of bias.

We stress that the photovoltage maximum occurs for perpendicular orientation of the light polarization plane with respect to the potential barrier, when the majority of photoexcited electrons are moving parallel to the junction. This is due to the driving operator in the kinetic equation (eq 4) which acts on the $\theta$-dependent steady state distribution function in a nontrivial way. The $\theta$-dependent part of the driving operator can be written as

$$
\begin{aligned}
\left(\mathbf{F} \cdot \nabla_{\mathbf{p}}\right)_{\theta} & =\left(-F_{x} p_{y}+F_{y} p_{x}\right) \frac{1}{p^{2}} \frac{\partial}{\partial \theta} \\
& =\sin (\phi-\theta) \frac{F}{p} \frac{\partial}{\partial \theta}
\end{aligned}
$$

where the relations $\left(\partial \theta / \partial p_{x}\right)=-p_{y} / p^{2}$ and $\left(\partial \theta / \partial p_{y}\right)=p_{x} / p^{2}$ have been utilized. Thus, the driving operator acting on the function of $\theta$ is maximum when the force and direction of particle motion are perpendicular, i.e., $\phi-\theta=\pi / 2$. Then, the major contribution to $\Delta f_{ \pm}$comes from electrons moving parallel to the barrier, photogenerated by the polarized light with $\theta_{\mathrm{pol}}=0^{\circ}$, as shown in Figure 10a,b. The maximum photovoltage occurs therefore at $\theta_{\mathrm{pol}}=0^{\circ}$, not at $\theta_{\mathrm{pol}}=90^{\circ}$, as one might expect. A similar $90^{\circ}$ offset was found in the photocurrent calculations of ref 33 .

We distinguish two cases of $n-n$ and $p-n$ graphene junctions. The former is simpler, and the resulting $\cos ^{2} \theta_{\mathrm{pol}}{ }^{-}$ dependent photovoltage term reads:

$$
\begin{gathered}
-q V_{\mathrm{osc}}^{\mathrm{ph}}\left(\theta_{\mathrm{pol}}\right)=\cos ^{2} \theta_{\mathrm{pol}} \frac{\tau_{1} \lambda^{2} v_{\mathrm{F}}^{2}}{2 \pi c^{2}} \frac{W_{\mathrm{a}}}{\pi d^{2} / 4} \\
\times \ln \left[\frac{\mu_{0}-U\left(x+\frac{d}{2}\right)}{\mu_{0}-U\left(x-\frac{d}{2}\right)}\right]
\end{gathered}
$$

Here, $\lambda$ is the light wavelength, $\mu_{0}$ is the chemical potential in graphene in the absence of top metallic contacts, $U(x)$ is the built-in potential profile due to the metallic contacts, and the laser spot diameter is $d=1.5 \mathrm{~mm}$. To simplify the expression, we assume $\left[\mu_{0}-U(x)\right] \gg T$ for any $x$, with $x$ the laser spot position. The absorbed radiation energy is characterized by the absorbed power $W_{\mathrm{a}}=\left(\pi e^{2} / \hbar c\right) W_{\mathrm{i}}$, which depends on the incident radiation power $W_{\mathrm{i}}$, and SLG optical absorption $\left(\pi e^{2}\right)$ $\hbar c)=0.023$. If the laser beam is focused on the middle of the $\mathrm{n}-\mathrm{n}$ junction at $x=0$ and its size is larger than the junction region, then $\Delta U=U(d / 2)-U(-d / 2)$ is the built-in potential step forming the junction. The photovoltage depends weakly on $\Delta U$ and the logarithmic multiplier is smaller than 1 for $\Delta U$ of a few tens to hundreds of $\mathrm{meV}$, satisfying the $\left[\mu_{0}-U(x)\right] \gg$ $T$ criterion. This behavior is different for the $\mathrm{p}-\mathrm{n}$ junctions shown in Figure 1, where $\left[\mu_{0}-U(x)\right] \ll T$ in the middle of the junction.

In what follows we assume $\mu_{0}=0$ and the electrochemical potential characterized by $U(x)$ alone. Equation 6 is then rewritten as

$$
\begin{gathered}
-q V_{\mathrm{osc}}^{\mathrm{ph}}\left(\theta_{\mathrm{pol}}\right)=\cos ^{2} \theta_{\mathrm{pol}} \frac{\tau_{1} \lambda^{2} v_{\mathrm{F}}^{2}}{2 \pi c^{2}} \frac{W_{\mathrm{a}}}{\pi d^{2} / 4} \\
\times \int_{U\left(x-\frac{d}{2}\right) / 2 T}^{U\left(x+\frac{d}{2}\right) / 2 T} \frac{\mathrm{d} \xi}{\ln (2 \cosh \xi)}
\end{gathered}
$$

$T$ appears in eq 7 since the condition $U(x) \gg T$ utilized before cannot apply in the middle of the $\mathrm{p}-\mathrm{n}$ junction, where $U=0$ (Figure 1). Moreover, we neglect the spatial dependence $T(x)$ here because it is overwhelmed by the much stronger dependence $U(x)$. At $x=0$ (i.e., laser spot in the middle of the junction) and $(U( \pm d / 2) / 2 T)=1$ (i.e., a potential step of $100 \mathrm{meV}$ ), the integral is $\sim 1$. At a radiation power of $300 \mu \mathrm{W}$ and $\lambda=633 \mathrm{~nm}$, the amplitude of $\cos ^{2} \theta_{\text {pol }}$ oscillations is a few microVolts. The photovoltage amplitude $\Delta V_{\mathrm{osc}}^{\mathrm{ph}}$ at $x=0$ is then given by

$$
\Delta V_{\mathrm{osc}}^{\mathrm{ph}}\left(\theta_{\mathrm{pol}}\right)=\frac{\tau_{1} \lambda^{2} v_{\mathrm{F}}^{2}}{2 \pi|q| c^{2}} \frac{W_{a}}{\pi d^{2} / 4} \times \int_{U\left(-\frac{d}{2}\right) / 2 T}^{U\left(+\frac{d}{2}\right) / 2 T} \frac{\mathrm{d} \xi}{\ln (2 \cosh \xi)}
$$

This is our main theoretical outcome and is computed for our device as a function of $\lambda$ in Figure 11 .

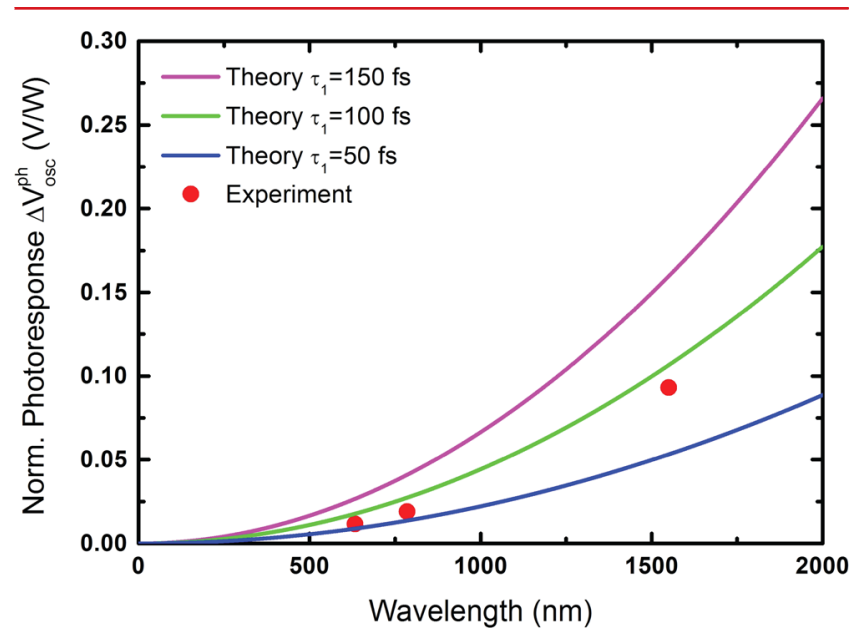

Figure 11. Experimental and theoretical polarization-dependent photovoltage amplitude $\Delta V_{\text {osc }}^{\mathrm{ph}}$ for our graphene $\mathrm{p}-\mathrm{n}$ junction as a function of incident light wavelength. Equation 8 is used with the following parameters: $\tau_{1}=150,100$, and $50 \mathrm{fs}, v_{\mathrm{F}}=10^{6} \mathrm{~ms}^{-1}, d=1.5$ $\mathrm{mm}, U(-d / 2)=-100 \mathrm{meV}, U(+d / 2)=50 \mathrm{meV}, T=25 \mathrm{meV}$.

Note that the photovoltage is higher for longer wavelengths. This is because light with a longer wavelength, but the same radiation power, can excite more electrons into the conduction band, resulting in a stronger photoresponse. $V^{\mathrm{ph}}$ also contains a polarization-independent term of the same order as $V_{\mathrm{osc}}^{\mathrm{ph}}$. It is however not possible to separate this term from the thermoelectric contributions, which are isotropic and independent of the incoming light polarization, due to the isotropy of graphene and the Au film; see last section of Methods. The 
oscillating, incident light polarization dependent part of Figure 7 is thus a direct proof of a photoelectric contribution to the overall generated photovoltage. The magnitude of these oscillations with respect to the overall photoresponse allows us to estimate the relative photoelectric contribution $P_{\mathrm{pe}}$ to the overall photoresponse. Figure 7 shows that $P_{\mathrm{pe}}$ is at least 21, 25, and $53 \%$ for 633,785 , and $1550 \mathrm{~nm}$. The experimentally and theoretically determined amplitude of the photoelectric polarization dependent part $\Delta V_{\mathrm{osc}}^{\mathrm{ph}}$ is shown in Figure 11, which demonstrates an increase of photoelectric contributions toward longer wavelengths.

In conclusion, we demonstrated the influence of the orientation of the lateral $\mathrm{p}-\mathrm{n}$ junction in graphene-based photodetectors with respect to the polarization of incident linearly polarized light. The angular dependence is in good agreement with theory and a proof that both photothermoelectric and photoelectric effects contribute to the photoresponse in MGM-photodetectors, with photoelectric effects becoming more pronounced at longer wavelengths. Further, we demonstrated that the light generated anisotropic distribution of carriers in momentum space can be observed in electrical measurements despite their relaxation on ultrafast time scales $\left(\tau_{1}\right)$. This might open the possibility for graphene-based photodetectors that can detect incident light and its polarization on ultrafast time scales, overcoming the thus-far speed limiting time constant $\tau_{2}$.

Methods. Kinetic Equation for Optically Excited Carriers in Graphene with a Built-in Potential $U(x)$. The Boltzmann kinetic eq 4 introduced above for $\Delta f_{ \pm}=f_{ \pm}-f_{ \pm}^{(0)}$ can be written as

$$
-q \frac{\partial V}{\partial x} \frac{\partial f_{ \pm}}{\partial p_{x}}-\frac{\partial U}{\partial x} \frac{\partial f_{ \pm}}{\partial p_{x}}+v_{ \pm} \frac{\partial f_{ \pm}}{\partial x}=g_{ \pm}^{\mathrm{ph}}-\frac{f_{ \pm}-f_{ \pm}^{(0)}}{\tau_{1}}
$$

where $p_{x}=\hbar k_{x}, v_{ \pm}= \pm v_{\mathrm{F}} k_{x} / k, q=-|q|$ is the electron charge, and $U(x)$ is the built-in potential. The bias voltage is assumed to be small enough to justify the linear response in terms of $(\partial V / \partial x)$. In the absence of bias and photogeneration, the system is in the equilibrium state described by the Fermi-Dirac distribution function $f_{ \pm}^{(0)}$ :

$$
f_{ \pm}^{(0)}=\frac{1}{1+\exp \left[\left( \pm \hbar v_{\mathrm{F}} k+U(x)-\mu_{0}\right) / k_{B} T\right]}
$$

" \pm " stands for the conduction and valence band. The photogeneration rate $g_{ \pm}^{\mathrm{ph}}$ can be derived from Fermi's golden rule using the unperturbed eigenstates of $H_{0}, \psi_{ \pm \mathrm{k}}(x, y)=(1 /$ $\sqrt{ } 2) \mathrm{e}^{i k_{x} x+i k_{y} y}\left(1, \pm \mathrm{e}^{i \theta}\right)^{T}$, where $\tan \theta=k_{y} / k_{x}$. For a given spin/ valley channel we get:

$$
\begin{aligned}
g_{ \pm}^{\mathrm{ph}}= & \frac{2 \pi}{\hbar}\left(\frac{\hbar q v_{\mathrm{F}} E_{0}}{2 \hbar \omega}\right)^{2} \sin ^{2}\left(\theta_{\mathrm{pol}}-\theta\right) \times \delta\left(\hbar \omega-2 \hbar v_{\mathrm{F}} k\right) \\
& \times\left(f_{\mp}^{(0)}-f_{ \pm}^{(0)}\right)
\end{aligned}
$$

where $\theta_{\mathrm{pol}}$ is the polarization angle, and $E_{0}$ is the electric field amplitude of the electromagnetic wave, which can be related to the incident radiation power per unit square as $w_{\mathrm{i}}=(c / 4 \pi)\langle[\mathbf{E}$ $\left.\times \mathbf{B}]_{z}\right\rangle_{t}=\left(c E_{0}^{2} / 8 \pi\right)\left[\mathrm{W} / \mathrm{cm}^{2}\right]$. The fluence can be estimated as $\Phi=w_{\mathrm{i}} \Delta t$. The integral radiation power is $W_{\mathrm{i}}=w_{\mathrm{i}}\left(\pi d^{2} / 4\right)$, where $d$ is the laser spot diameter.

We look for the solution of eq 9 in the form $f_{ \pm}=f_{ \pm}^{(0)}+f_{ \pm}^{\text {h }}+$ $f_{ \pm}^{(1)}$, where $f_{ \pm}^{\mathrm{ph}}=\tau_{1} g_{ \pm}^{\mathrm{ph}}$, and $f_{ \pm}^{(1)}$ is determined from the following equation obtained substituting $f_{ \pm}$in eq 9:

$$
\begin{gathered}
-q \frac{\partial V}{\partial x} \frac{\partial f_{ \pm}^{(0)}}{\partial p_{x}}-\frac{\partial U}{\partial x} \frac{\partial f_{ \pm}^{(0)}}{\partial p_{x}}+v_{ \pm} \frac{\partial f_{ \pm}^{(0)}}{\partial x}-q \frac{\partial V}{\partial x} \frac{\partial f_{ \pm}^{\mathrm{ph}}}{\partial p_{x}} \\
-\frac{\partial U}{\partial x} \frac{\partial f_{ \pm}^{\mathrm{ph}}}{\partial p_{x}}+v_{ \pm} \frac{\partial f_{ \pm}^{\mathrm{ph}}}{\partial x}=-\frac{f_{ \pm}^{(1)}}{\tau_{1}}
\end{gathered}
$$

One can prove directly that $-(\partial U / \partial x)\left(\partial f_{ \pm}^{(0)} / \partial p_{x}\right)+v_{ \pm}\left(\partial f_{ \pm}^{(0)} /\right.$ $\partial x)=0$. Moreover, $-q(\partial V / \partial x)\left(\partial f_{ \pm}^{\mathrm{ph}} / \partial p_{x}\right) \ll-q(\partial V / \partial x)\left(\partial f_{ \pm}^{ \pm 0} /\right.$ $\left.\partial p_{x}\right)$ since $n^{\mathrm{ph}} \ll n$, as discussed in the main text. Taking into account the $\theta$ dependence of $f_{ \pm}^{\mathrm{ph}}, f_{ \pm}^{(1)}$ can be written as

$$
\begin{aligned}
& \frac{f_{ \pm}^{(1)}}{\tau_{1}}=q \frac{\partial V}{\partial x} \frac{\partial f_{ \pm}^{(0)}}{\partial p_{x}}+\frac{\partial U}{\partial x} \frac{\pi \tau_{1} v_{\mathrm{F}}}{2 \hbar}\left(\frac{q E_{0}}{2 \hbar \omega}\right)^{2}\left(f_{\mp}^{(0)}-f_{ \pm}^{(0)}\right) \\
& \quad \times\left\{\cos \theta\left[1-\cos \left(2 \theta_{\mathrm{pol}}-2 \theta\right)\right]\left[\frac{\partial}{\partial k} \delta\left(k-\frac{\omega}{2 v_{\mathrm{F}}}\right)\right]\right. \\
& \left.\quad+2 \sin \theta \sin \left(2 \theta_{\mathrm{pol}}-2 \theta\right) \frac{1}{k} \delta\left(k-\frac{\omega}{2 v_{\mathrm{F}}}\right)\right\}
\end{aligned}
$$

Note the graphene specific contribution proportional to $\left(\partial \sin ^{2}\left(\theta-\theta_{\text {pol }}\right)\right) / \partial k_{x}=\sin \left(2 \theta_{\text {pol }}-2 \theta\right)(\sin \theta) /(k)$. To calculate the current density we multiply eq 13 by $v_{ \pm}$and integrate it over $k$ and $\theta$. We take $f_{+}^{(0)}\left(k=\omega / 2 v_{\mathrm{F}}\right)=0$ and $f_{-}^{(0)}(k$ $\left.=\omega / 2 v_{\mathrm{F}}\right)=1$, a reasonable assumption for any $T$, electrochemical doping and wavelength we consider in this paper. In order to find the photovoltage for the open circuit we employ in our measurements, the total current density and external bias are set to zero. In this case, $V$ in eq 13 is the photovoltage $V^{\text {ph }}$.

Photoresponse of Graphene $n-n$ Junction. Here we assume that $\left[\mu_{0}-U(x)\right] \gg T$, so that eq 13 can be integrated:

$$
\begin{aligned}
& q \frac{\partial V^{\mathrm{ph}}}{\partial x}\left(\mu_{0}-U(x)\right)+\left(2+\cos 2 \theta_{\mathrm{pol}}\right) \frac{\partial U}{\partial x} \frac{\pi \tau_{1}}{2 \hbar}\left(\frac{q v_{\mathrm{F}} E_{0}}{2 \omega}\right)^{2} \\
& \quad=0
\end{aligned}
$$

To obtain $V^{\text {ph }}$ we integrate eq 14 over $x$ within the laser spot:

$$
\begin{aligned}
-q V^{\mathrm{ph}}= & \left(2+\cos 2 \theta_{\mathrm{pol}}\right) \frac{\pi \tau_{1}}{2 \hbar}\left(\frac{q v_{\mathrm{F}} E_{0}}{2 \omega}\right)^{2} \times \int_{x-d / 2}^{x+d / 2} \mathrm{~d} x \\
& \frac{1}{\mu_{0}-U(x)} \frac{\partial U}{\partial x}
\end{aligned}
$$

The $E_{0}$-dependent multiplier in eq 15 can be expressed in terms of the absorbed radiation power $W_{\mathrm{a}}=\left(\pi e^{2} / \hbar c\right) W_{\mathrm{i}}$. Extracting the $\theta_{\text {pol }}$ dependent part out of eq 15 , we arrive at the final result of eq 6.

Photoresponse of the Graphene $p-n$ Junction. Here the electrochemical potential can be smaller than $T$ in the junction region and we cannot assume $\left[\mu_{0}-U(x)\right] \gg T$. To simplify, we set $\mu_{0}=0$, so that the electrochemical potential is determined by $U(x)$ alone. Note that eq 14 is now $T$ dependent:

$$
\begin{gathered}
q \frac{\partial V^{\mathrm{ph}}}{\partial x} 2 T \ln \left[2 \cosh \frac{U(x)}{2 T}\right]+\left(2+\cos 2 \theta_{\mathrm{pol}}\right) \frac{\partial U}{\partial x} \\
\frac{\pi \tau_{1}}{2 \hbar} \times\left(\frac{q v_{\mathrm{F}} E_{0}}{2 \omega}\right)^{2}=0
\end{gathered}
$$

and the photovoltage becomes 


$$
\begin{aligned}
-q V^{\mathrm{ph}}= & \left(2+\cos 2 \theta_{\mathrm{pol}}\right) \frac{\pi \tau_{1}}{2 \hbar}\left(\frac{q v_{\mathrm{F}} E_{0}}{2 \omega}\right)^{2} \times \\
& \int_{x-d / 2}^{x+d / 2} \frac{\mathrm{d} x}{2 T} \frac{1}{\ln \left[2 \cosh \frac{U(x)}{2 T}\right]} \frac{\partial U}{\partial x}
\end{aligned}
$$

One can exclude $T$ from the integrand. The final formula for $V^{\text {ph }}$ reads:

$$
\begin{aligned}
-q V^{\mathrm{ph}}= & \left(2+\cos 2 \theta_{\mathrm{pol}}\right) \frac{\pi \tau_{1}}{2 \hbar}\left(\frac{q v_{\mathrm{F}} E_{0}}{2 \omega}\right)^{2} \times \\
& \int_{-U(x-d / 2) / 2 T}^{U(x+d / 2) / 2 T} \frac{\mathrm{d} \xi}{\ln (2 \cosh \xi)}
\end{aligned}
$$

Extracting the $\theta_{\text {pol }}$ dependent part from eq 18, we get eq 7 .

Thermoelectric Contribution in the Total Photoresponse. An irradiated sample experiences heating, therefore the electrons are subject to a $T$ gradient $(\partial T / \partial x)$, which appears in eq 9 , when $v_{ \pm}\left(\partial f_{ \pm} / \partial x\right)$ is written explicitly. Following the same procedure as above, we arrive at eq 12 , where $-(\partial U /$ $\partial x)\left(\partial f_{ \pm}^{(0)} / \partial p_{x}\right)+v_{ \pm}\left(\partial f_{ \pm}^{(0)} / \partial x\right)$ is not zero and gives the leading contribution in terms of $(\partial T / \partial x)$. This cannot depend on light polarization in any circumstance.

For eq 14, the thermoelectric term can be estimated as $\left(\pi^{2}\right)$ 3) $T(x)(\partial T / \partial x)$, which results in the photothermoelectric term given by eq 2 . Thus, the thermoelectric contribution, being proportional to $T$, gets larger for hot electrons and becomes dominant in this case. Most importantly, the thermoelectric response mainly depends on the radiation power converted to heat and is not sensitive to any particular light polarization. In contrast, the photoelectric response (eq 15) does depend on the polarization angle $\theta_{\mathrm{pol}}$, which makes it possible to separate these two effects in the total response measured. Note, however, that the photovoltage (eq 15) also contains a $\theta_{\mathrm{pol}}$ independent contribution, which is not possible to distinguish from the thermoelectric response. Nevertheless, the amplitude of $\cos 2 \theta_{\text {pol }}$ oscillations gives indication of how large the photoelectric response is.

\section{AUTHOR INFORMATION}

\section{Corresponding Author}

*E-mail: acf26@eng.cam.ac.uk.

\section{Notes}

The authors declare no competing financial interest.

\section{ACKNOWLEDGMENTS}

We thank Andrey Klots for useful discussions. We acknowledge funding from EU Graphene Flagship (contract no.604391), ERC grants NANOPOTS, Hetero2D, a Royal Society Wolfson Research Merit Award, EU projects GENIUS, CARERAMM, EPSRC grants EP/K01711X/1, EP/K017144/1, EP/L016087/ 1, Nokia Research Centre Cambridge, Deutsche Forschungsgemeinschaft (DFG) GRK 1570 and SPP 1285.

\section{REFERENCES}

(1) Bonaccorso, F.; Sun, Z.; Hasan, T.; Ferrari, A. C. Nat. Photonics 2010, 4, 611-622.

(2) Bae, S.; Kim, H.; Lee, Y.; Xu, X.; Park, J.-S.; Zheng, Y.; Balakrishnan, J.; Lei, T.; Ri Kim, H.; Song, Y. I.; Kim, Y.-J.; Kim, K. S.; Özyilmaz, B.; Ahn, J.-H.; Hong, B. H.; Iijima, S. Nat. Nanotechnol. 2010, 5, 574-578.

(3) de Arco, L. G.; Zhang, Y.; Schlenker, C. W.; Ryu, K.; Thompson, M. E.; Zhou, C. ACS Nano 2010, 5, 2865-2873.
(4) Liu, M.; Yin, X.; Ulin-Avila, E.; Geng, B.; Zentgraf, T.; Ju, L.; Wang, F.; Zhang, X. Nature 2011, 474, 64-67.

(5) Ju, L.; Geng, B.; Horng, J.; Girit, C.; Martin, M.; Hao, Z.; Bechtel, H. A.; Liang, X.; Zettl, A.; Shen, Y. R.; Wang, F. Nat. Nanotechnol. 2011, 6, 630-634.

(6) Echtermeyer, T. J.; Britnell, L.; Jasnos, P. K.; Lombardo, A.; Gorbachev, R. V.; Grigorenko, A. N.; Geim, A. K.; Ferrari, A. C.; Novoselov, K. S. Nat. Commun. 2011, 2, 458.

(7) Schedin, F.; Lidorikis, E.; Lombardo, A.; Kravets, V. G.; Geim, A. K.; Grigorenko, A. N.; Novoselov, K. S.; Ferrari, A. C. ACS Nano 2010, 4, 5617-5626.

(8) Fei, Z.; et al. Nature 2012, 487, 82-85.

(9) Chen, J.; et al. Nature 2012, 487, 77-81.

(10) Engel, M.; Steiner, M.; Lombardo, A.; Ferrari, A. C.; v. Löehneysen, H.; Avouris, P.; Krupke, R. Nat. Commun. 2012, 3, 906.

(11) Furchi, M.; Urich, A.; Popischil, A.; Lilley, G.; Unterrainer, K.; Detz, H.; Klang, P.; Andrews, A. M.; Schrenk, W.; Strasser, G.; Mueller, T. Nano Lett. 2012, 12, 2773-2777.

(12) Sun, Z.; Hasan, T.; Torrisi, F.; Popa, D.; Privitera, G.; Wang, F.; Bonaccorso, F.; Basko, D. M.; Ferrari, A. C. ACS Nano 2010, 4, 803810.

(13) Lee, E. J. H.; Balasubramanian, K.; Weitz, R. T.; Burghard, M.; Kern, K. Nat. Nanotechnol. 2008, 3, 486-490.

(14) Mueller, T.; Xia, F.; Freitag, M.; Tsang, J.; Avouris, P. Phys. Rev. B 2009, 79, 245430.

(15) Park, J.; Ahn, Y. H.; Ruiz-Vargas, C. Nano Lett. 2009, 9, $1742-$ 1746.

(16) Mueller, T.; Xia, F.; Avouris, P. Nat. Photonics 2010, 4, 297301.

(17) Urich, A.; Unterrainer, K.; Mueller, T. Nano Lett. 2011, 11, 2804-2808.

(18) Xia, F. N.; Mueller, T.; Lin, Y. M.; Valdes-Garcia, A.; Avouris, P. Nat. Nanotechnol. 2009, 4, 839-843.

(19) Lemme, M. C.; Koppens, F. H. L.; Falk, A. L.; Rudner, M. S.; Park, H.; Levitov, L. S.; Marcus, C. M. Nano Lett. 2011, 11, 41344137.

(20) Gabor, N. M.; Song, J. C. W.; Ma, Q.; Nair, N. L.; Taychatanapat, T.; Watanabe, K.; Taniguchi, T.; Levitov, L. S.; Jarillo-Herrero, P. Science 2011, 334, 648-652.

(21) Xu, X. D.; Gabor, N. M.; Alden, J. S.; van der Zande, A. M.; McEuen, P. L. Nano Lett. 2010, 10, 562-566.

(22) Konstantatos, G.; Badioli, M.; Gaudreau, L.; Osmond, J.; Bernechea, M.; Pelayo Garcia de Arquer, F.; Gatti, F.; Koppens, F. H. L. Nat. Nanotechnol. 2012, 7, 363-368.

(23) Freitag, M.; Low, T.; Zhu, W.; Yan, H.; Xia, F.; Avouris, P. Nat. Commun. 2013, 4, 1951.

(24) Yan, H.; Low, T.; Zhu, W.; Wu, Y.; Freitag, M.; Li, X.; Guinea, F.; Avouris, P.; Xia, F. Nat. Photonics 2013, 7, 394-399.

(25) Pospischil, A.; Humer, M.; Furchi, M. M.; Bachmann, D.; Guider, R.; Fromherz, T.; Mueller, T. Nat. Photonics 2013, 7, 892896.

(26) Liu, Y.; Cheng, R.; Liao, L.; Zhou, H.; Bai, J.; Liu, G.; Liu, L.; Huang, Y.; Duan, X. Nat. Commun. 2011, 2, 579.

(27) Kim, K.; Choi, J.-Y.; Kim, T.; Cho, S.-H.; Chong, H.-J. Nature 2011, 479, 338-344.

(28) Vicarelli, L.; Vitiello, M. S.; Coquillat, D.; Lombardo, A.; Ferrari, A. C.; Knap, W.; Polini, M.; Pellegrini, V.; Tredicucci, A. Nat. Mater. 2012, 11, 865-871.

(29) Spirito, D.; Coquillat, D.; De Bonis, S. L.; Lombardo, A.; Bruna, M.; Ferrari, A. C.; Pellegrini, V.; Tredicucci, A.; Knap, W.; Vitiello, M. S. arXiv: 1312.3737, 2013.

(30) Giovannetti, G.; Khomyakov, P. A.; Brocks, G.; Karpan, V. M.; van den Brink, J.; Kelly, P. J. Phys. Rev. Lett. 2008, 101, 026803.

(31) Lemme, M. C.; Echtermeyer, T. J.; Baus, M.; Kurz, H. IEEE EDL 2007, 28, 282-284.

(32) Song, J. C. W.; Rudner, M. S.; Marcus, C. M.; Levitov, L. S. Nano Lett. 2011, 11, 4688-4692.

(33) Mai, S.; Syzranov, S. V.; Efetov, K. B. Phys. Rev. B 2011, 83, 033402 . 
(34) Ashcroft, N. W.; Mermin, N. D. Solid State Physics; Thomson Learning Inc.: New York, 1976.

(35) Zuev, Y. M.; Chang, W.; Kim, P. Phys. Rev. Lett. 2009, 102, 096807.

(36) Wei, P.; Bao, W.; Pu, Y.; Lau, C. N.; Shi, J. Phys. Rev. Lett. 2009, 102, 166808.

(37) Sze, S. M. Physics of Semiconductor Devices; Wiley Interscience: New York, 1981.

(38) Novoselov, K. S.; Geim, A. K.; Morozov, S. V.; Jiang, D.; Zhang, Y.; Dubonos, S. V.; Grigorieva, I. V.; Firsov, A. A. Science 2004, 306, 666-669.

(39) Novoselov, K. S.; Jiang, D.; Schedin, F.; Booth, T. J.; Khotkevich, V.; Morozov, S. V.; Geim, A. K. Proc. Natl. Acad. Sci. U.S.A. 2005, 102, 10451-10453.

(40) Casiraghi, C.; Hartschuh, A.; Lidorikis, E.; Qian, H.; Harutyunyan, H.; Gokus, T.; Novoselov, K. S.; Ferrari, A. C. Nano Lett. 2007, 7, 2711-2717.

(41) Ferrari, A. C.; Meyer, J. C.; Scardaci, V.; Casiraghi, C.; Lazzeri, M.; Mauri, F.; Piscanec, S.; Jiang, D.; Novoselov, K. S.; Roth, S.; Geim, A. K. Phys. Rev. Lett. 2006, 97, 187401.

(42) Ferrari, A. C.; Basko, D. M. Nat. Nanotechnol. 2013, 8, 235-246.

(43) Cancado, L. G.; Jorio, A.; Martins Ferreira, E. H.; Stavale, F.; Achete, C. A.; Capaz, R. B.; Moutinho, M. V. O.; Lombardo, A.; Kulmala, T. S.; Ferrari, A. C. Nano Lett. 2011, 11, 3190-3196.

(44) Ferrari, A. C.; Robertson, J. Phys. Rev. B 2000, 61, 14095.

(45) Ferrari, A. C. Solid State Commun. 2007, 143, 47-57.

(46) Pisana, S.; Lazzeri, M.; Casiraghi, C.; Novoselov, K. S.; Geim, A. K.; Ferrari, A. C.; Mauri, F. Nat. Mater. 2007, 6, 198-201.

(47) Das, A.; Pisana, S.; Chakraborty, B.; Piscanec, S.; Saha, S. K.; Waghmare, U. V.; Novoselov, K. S.; Krishnamurthy, H. R.; Geim, A. K.; Ferrari, A. C.; Sood, A. K. Nat. Nanotechnol. 2008, 3, 210-215.

(48) Mak, K. F.; Sfeir, M. Y.; Wu, Y.; Lui, C. H.; Misewich, J. A.; Heinz, T. F. Phys. Rev. Lett. 2008, 101, 196405.

(49) Lagatsky, A. A.; et al. Appl. Phys. Lett. 2013, 102, 013113.

(50) Ermushev, A. V.; Mchedlishvili, B. V.; OleÄnikov, V. A.; Petukhov, A. V. Quantum Electron. 1993, 23, 435-440.

(51) Tritt, T. M. Thermal conductivity; Springer: New York, 2004.

(52) Balandin, A. A.; Ghosh, S.; Bao, W.; Calizo, I.; Teweldebrhan, D.; Miao, F.; Lao, C. N. Nano Lett. 2008, 8, 902-907.

(53) Chrisey, D. B.; Hubler, G. K. Pulsed Laser Deposition of Thin Films; John Wiley \& Sons: New York, 1994.

(54) Clarkson Kaye, G. W.; Laby, T. H. Tables of physical and chemical constants, 16th ed.; Longman: New York, 1995.

(55) Palik, E. D. Handbook of Optical Constants of Solids; Academic Press: Boston, 1985.

(56) Echtermeyer, T. J.; Wu, M.; Milana, S.; Sassi, U.; Lidorikis, E.; Ferrari, A. C. submitted (2014).

(57) McCann, E. Graphene Nanoelectronics: Metrology, Synthesis, Properties and Applications; Springer: Heidelberg, 2012.

(58) Brida, D.; et al. Nat. Commun. 2013, 4, 1987.

(59) George, P. A.; Strait, J.; Dawlaty, J.; Shivaraman, S.; Chandrashekhar, M.; Rana, F.; Spencer, M. G. Nano Lett. 2008, 8, 4248-4251.

(60) Dawlaty, J. M.; Shivaraman, S.; Chandrashekhar, M.; Rana, F.; Spencer, M. G. Appl. Phys. Lett. 2008, 92, 042116.

(61) Malic, E.; Winzer, T.; Knorr, A. Appl. Phys. Lett. 2012, 101, 213110.

(62) Malic, E.; Winzer, T.; Bobkin, E.; Knorr, A. Phys. Rev. B 2011, 84, 205406.

(63) Tomadin, A.; Brida, D.; Cerullo, G.; Ferrari, A. C.; Polini, M. Phys. Rev. B 2013, 88, 035430.

(64) Song, J. C. W.; Reizer, M. Y.; Levitov, L. S. Phys. Rev. Lett. 2012, 109, 106602.

(65) Graham, M. W.; Shi, S.-F.; Ralph, D. C.; Park, J.; McEuen, P. L. Nat. Phys. 2012, 9, 103-108.

(66) Breusing, M.; Kuehn, S.; Winzer, T.; Malić, E.; Milde, F.; Severin, N.; Rabe, J. P.; Ropers, C.; Knorr, A.; Elsaesser, T. Phys. Rev. B 2011, 83, 153410.
(67) Nair, R. R.; Blake, P.; Grigorenko, A. N.; Novoselov, K. S.; Booth, T. J.; Stauber, T.; Peres, N. M. R.; Geim, A. K. Science 2008, 320, 1308.

(68) Nelson, J. The Physics of Solar Cells; Imperial College Press: London, 2004. 